\title{
Confusion, Hyperactive Delirium, and Secondary Mania in Right Hemispheric Strokes: A Focused Review of Neuroanatomical Correlates
}

\author{
David L. Perez ${ }^{1,2,3,4 *}$, Eva Catenaccio ${ }^{4}$ and Jane Epstein ${ }^{3,4}$
}

${ }^{1}$ Department of Neurology, Brigham and Women's Hospital, Boston MA 02115

${ }^{2}$ Department of Neurology, Massachusetts General Hospital, Boston MA 02114

${ }^{3}$ Department of Psychiatry, Brigham and Women's Hospital, Boston MA 02115

${ }^{4}$ Functional Neuroimaging Laboratory, Brigham and Women's Hospital, Boston, MA 02115, USA

\begin{abstract}
Affective and behavioral neuropsychiatric disturbances are frequently encountered in post-stroke patients. While the neural correlates of conditions such as post-stroke depression and apathy have been well investigated, confusion, hyperactive delirium, and secondary mania are less well detailed. In this article, a case of right hemispheric stroke presenting with acute confusion and agitation is described to introduce the topic, followed by a focused review of the post-stroke right hemispheric lesional correlates of non-agitated confusion, hyperactive delirium, and secondary mania. These three post-stroke syndromes have both overlapping and non-overlapping structural components. Non-agitated confusional states may preferentially involve higher order perceptual/attentional association cortices (parietal, occipital, prefrontal) and related subcortical connections. Right hemispheric post-stroke patients with hyperactive delirium often share similar lesion sites, but also have a greater tendency to involve limbic related medial temporal lobe structures. Lesions particularly associated with secondary mania include orbitofrontal-subcortical circuits and medial temporal lobe structures.
\end{abstract}

Keywords: Post-stroke mania; Delirium; Agitation; Confusion

Abbreviations: CT: Computed Tomography; MCA: Middle Cerebral Artery; FLAIR: Fluid Attenuated Inversion Recovery; GPI: Globus Pallidus Interna; ${ }^{18}$ FDG PET: ${ }^{18}$ Flourodeoxyglucose Positron Emission Tomography; SPECT: Single-Positron Emission Computed Tomography

\section{Introduction}

Confusion is a neuropsychiatric term "denoting the patient's incapacity to think with customary speed, clarity, and coherence" and is associated with attentional and perceptional impairments [1]. A confusional state with agitation, or hyperactive delirium, is a related concept associated with increased psychomotor and autonomic activity in the setting of aberrant attentional and perceptual processes $[2,3]$. Trzepacz and colleagues have classified transient confusional states with or without agitation into distinct motoric subtypes of delirium: hyperactive, hypoactive and mixed [4]. As a whole, delirium is present in $36-50 \%$ of post-stroke patients [5,6], and one-half of those display psychomotor hyperactivity [7].

A less frequently encountered post-stroke disturbance is secondary mania. Secondary mania refers to persistently elevated, expansive or irritable mood with associated inflated self esteem, diminished need for sleep, talkativeness, racing thoughts (flight of ideas), distractibility, psychomotor agitation and/or excessive involvement in pleasurable activities in the setting of medical or neurologic illness [8,9]. Secondary mania, distinguished from hyperactive delirium by the presence of many of these features and the absence of attentional fluctuations, is a relatively rare disorder occurring in less than $1 \%$ of patients [1013]. Despite this epidemiology, non-agitated confusion, hyperactive delirium, and secondary mania when considered as a spectrum are frequently encountered neuropsychiatric symptoms on inpatient stroke services.

While motor, somatosensory, linguistic (aphasia), and visualspatial/attentional (neglect) deficits are readily appreciable manifestations of acute ischemic cerebrovascular accidents with identifiable structure-function relationships, affective and behavioral abnormalities have received less attention and may be masked by motor and linguistic deficits. Nevertheless, affective and behavioral symptoms provide localizing value, and in some instances are the most salient feature of a cerebrovascular event with minimal additional cortical or pyramidal tract signs [14]. While post-stroke symptoms including apathy, depression, and dysprosody have been intensely investigated [15-17], less well characterized are non-agitated confusion, hyperactive delirium, and mania, which are discussed in this focused review.

In this article, a case of ischemic right hemispheric stroke displaying acute confusion and agitation is described to introduce and illustrate the topic, and the right hemispheric neural correlates of nonagitated confusion, hyperactive delirium, and secondary mania are subsequently reviewed and discussed as sharing overlapping (and nonoverlapping) neural substrates in distributed cortical and subcortical brain networks mediating emotion and behavior.

\section{Illustrative Case}

A 76 year old, right-handed man presented to the emergency department with two-weeks of "strange" behavior as reported by his family. The patient exhibited varying levels of psychomotor activity with periods of hypo and hyperactivity, disorientation to place and time, and periods of uncharacteristic and unprovoked agitation. On the day prior to admission, he was noted by another family member

*Corresponding author: David L. Perez M.D, Brigham and Women's Hospital, Departments of Neurology and Psychiatry, 75 Francis Street, Boston, MA 02115, Tel: 551-427-1439; Fax: 617-732-6083; E-mail: david.Iperez@gmail. com

Received June 01, 2011; Accepted July 15, 2011; Published September 20, 2011

Citation: Perez DL, Catenaccio E, Epstein J (2011) Confusion, Hyperactive Delirium, and Secondary Mania in Right Hemispheric Strokes: A Focused Review of Neuroanatomical Correlates. J Neurol Neurophysiol S1. doi:10.4172/2155-9562. S1-003

Copyright: $\odot 2011$ Perez DL, et al. This is an open-access article distributed under the terms of the Creative Commons Attribution License, which permits unrestricted use, distribution, and reproduction in any medium, provided the original author and source are credited. 
to also have a mild left facial droop. His past medical history was notable for poorly controlled diabetes mellitus type II, hypertension, hyperlipidemia, and no previously recognized cognitive decline or mental health disturbance. His home medications included oral hypoglycemics and anti-hypertensives without use of anti-platelet or lipid lowering agents.

On presentation, he was afebrile with a blood pressure of 163/93. His physical examination was unremarkable. During bedside mental status testing, he was awake and agitated with profound psychomotor hyperactivity. He attempted to hit the interviewer in the face during questioning on several occasions. At other moments, he was inexplicably jovial and laughed spontaneously. He was disoriented to month, year and hospital name, and was unable to list the months of the year backwards. His receptive and expressive language skills were preserved, and he was without appreciable visual-spatial neglect. His neurologic examination revealed a very mild left hemi-body weakness with a facial droop and $-5 / 5$ upper motor neuron pattern of weakness in his arm and leg. He extinguished to double simultaneous light touch on the left, but had no primary somatosensory deficit. His left toe was extensor.

Admission labs showed no appreciable toxic-metabolic abnormality but were notable for a Troponin-T elevation of 0.20 with corresponding inferior Q-waves on electrocardiogram. Computed tomography (CT) neuroimaging revealed extensive bilateral periventricular and subcortical hypoattenuation suggestive of remote small vessel ischemia, right greater than left focal supraclinoid internal carotid artery stenosis on CT angiography, and increased mean transit time in the territory of the right middle cerebral artery (MCA) on CT perfusion. Magnetic resonance imaging showed multiple scattered small foci of restricted diffusion in the right MCA territory with corresponding fluid attenuated inversion recovery (FLAIR) increased signal; in addition, diffuse sulcal widening consistent with mild global atrophy was noted (Figure 1). Transthoracic echocardiogram revealed inferior and posterior left ventricle hypokinesis without evidence of intracardiac thrombus. 24-hour cardiac monitoring showed sinus rhythm. Right fronto-parietal slowing without epileptiform activity was seen on electroencephalogram. His low density lipoprotein was 114 and his hemoglobin H1c was 10.2. His stroke was deemed likely to be secondary to low flow (stenosis) and/or embolism from his calcified right supraclinoid internal carotid artery, and he was started on aspirin, a HMG-CoA reductase inhibitor (Statin), and quetiapine for intermittent agitation with good response.

\section{Review and Discussion}

\section{Confusional states with and without agitation}

Early post-mortem and more recent neuroimaging based studies examining structure-function relationships in acute right hemispheric stroke patients suggest distributed neuroanatomical substrates for lesion-related behavioral and affective disturbances. In a series of 9 patients with unilateral or bilateral posterior cerebral artery infarcts who abruptly developed agitation, inattention, disorientation, restlessness, and hallucinations, post-mortem examinations revealed hippocampal, ventral temporo-occipital and thalamic infarcts [18]. Additional early post-mortem investigations in a woman who developed a hyperactive delirium following an anterior communicating artery aneurysm rupture showed infarcts in bilateral anterior cingulate, orbitofrontal, right amygdala and hippocampus [19].

Subsequent neuroimaging studies further localized lesions in right hemispheric post-stroke patients with acute confusional states.
Mesulam and colleagues in 1976 identified 3 patients presenting acutely with confusion and agitation following right MCA infarcts [20]. These patients had lesions in parietal-occipital cortex (with concurrent mild cerebral atrophy), temporoparietal cortex, and the inferior frontal gyrus respectively. Persistent confusion and agitation has also been reported in 3 patients with right MCA infarcts despite resolution of motor deficits [21]. In a larger series of 308 inpatients screened for acute confusional states in the context of neurologic consultation, 19\% had inattention and distractibility [22]. Of those, 26 of 60 displayed radiographic evidence of focal lesions, with parietal and, to a lesser extent, adjacent temporal and occipital regions detected as the most common ischemic sites. Interestingly, patients with behavioral changes may exhibit minimal additional lateralizing or localizing signs as described previously with temporoparietal/posterior temporal infarcts [14]. In addition to cortical sites, subcortical lesions including caudate [23] and thalamic infarcts [24] may also manifest acutely with hyperactive delirium. More recently, 216 post-stroke patients were prospectively evaluated for confusion, with or without hyperactivity; a trend was noted for right hemispheric lesions to be associated with acute confusional states, particularly right MCA, caudate, and thalamic infarcts. Neglect (a non-dominant hemisphere sign) was independently associated with confusion [7].

Some studies have helped differentiate the right hemisphere neural correlates of non-agitated confusion with those of hyperactive delirium. In a study of 41 patients with acute right MCA infarcts, $61 \%$ exhibited confusion and 15\% displayed additional agitation [25]. Nonagitated confusion was associated with fronto-striatal lesions while hyperactive delirium was more common in patients with temporal lesions, suggesting that confusion and agitation may have dissociable
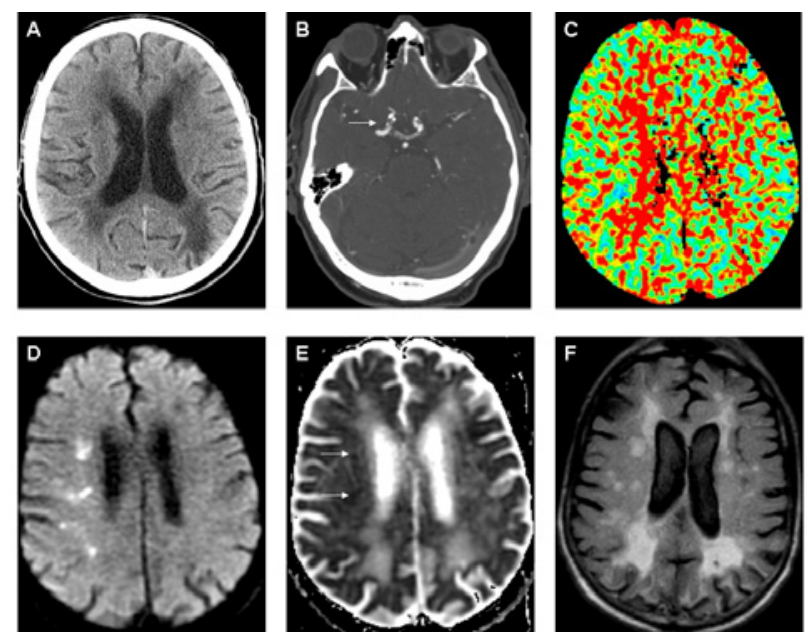

Figure 1: A. Non-contrast computed tomography (CT) scan showed bilateral periventricular and subcortical hypodensities suggestive of remote small vessel ischemia. An ill-defined right frontal subcortical hypodensity and an old left posterior parietal infarct were also appreciated. B. CT angiography identified bilateral right greater than left supraclinoid calcified plaque with moderate to severe right-sided stenosis (white arrow). C. CT perfusion scan showed prolonged mean transit time and mild increased cerebral blood volume (not shown) in the right subcortical middle cerebral artery territory. $D-E$. Diffusion weighted imaging and apparent diffusion coefficient scans showed restricted diffusion (arrows) in right subcortical fronto-parietal regions F. Axial fluid attenuated inversion recovery (FLAIR) imaging revealed bihemispheric periventricular and subcortical increased signal, diffuse sulcal widening with mild global atrophy, associated encephalomalacia in left posterior parietal region, and right subcortical increased signal corresponding to some of the restricted diffusion sites. Note: all images displayed using radiographic convention. 


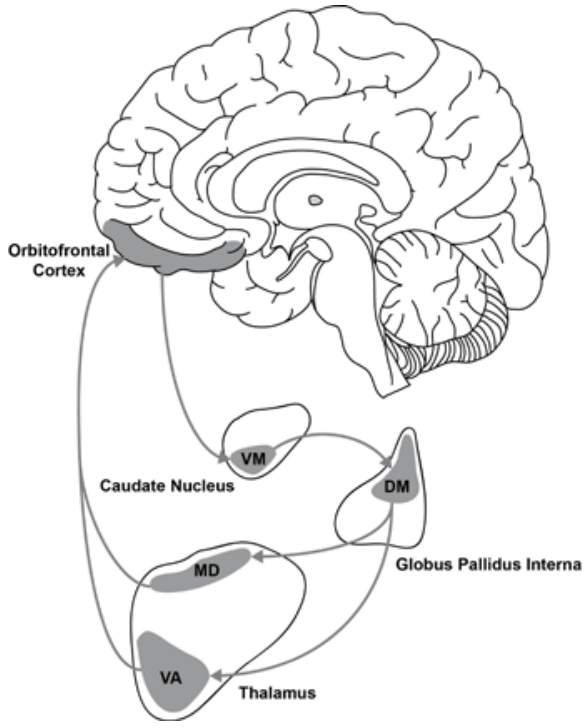

Figure 2: Orbitofrontal-subcortical circuit involved in empathic, socially appropriate behavior and implicated in secondary mania. VM indicates ventromedial; DM, dorsomedial; MD, mediodorsal; VA, ventral anterior.

neural substrates that frequently co-exist in right MCA infarcts. Further anatomical specificity was provided by an observational study of 10 patients with acute, isolated inferior (posterior) division, right MCA infarcts; 6 patients presented with agitated behavior, 5 with lesions in right temporoparietal regions and one with a posterior temporal infarct [26]. In a case further supporting the dissociation of neural substrates mediating confusion and hyperactive delirium, C. Miller Fisher in 1982 described a patient with persistent isolated disorientation for place, visual-spatial neglect without agitation, and a right inferior parietal-occipital ischemic stroke [27]. Thus, in limited studies, right hemispheric infarcts in posterior parietal, temporal, occipital, prefrontal, and subcortical lesion sites have been implicated in poststroke confusion and hyperactive delirium. Evidence further suggests that non-agitated confusional states in patients with right hemispheric strokes may be preferentially associated with lesions in attentional and perceptual association cortices (prefrontal, posterior parietal, occipital) and interconnected subcortical regions, while hyperactive delirium may involve additional limbic and paralimbic regions $[19,20,14]$.

\section{Secondary mania}

Right hemispheric ischemic lesions have also been associated with secondary mania. Robinson and coworkers in a series of early studies reported an association between acute right hemisphere stroke and undue cheerfulness $[15,28]$. In this series, 5 of 6 patients classified as hypomanic had right anterior lesions [15]. In subsequent anatomical investigations, cortical (predominantly orbitofrontal and medial temporal cortices) and subcortical right hemispheric lesions were implicated in secondary mania. Cummings and Mendez reported 2 patients with ventral thalamic infarcts who presented acutely with mania [29]. Of 4 right hemispheric stroke patients detailed by Starkstein and colleagues who exhibited manic symptoms and were subsequently imaged within one month of their affective disturbances, 2 had lesions in thalamocapsular regions, while temporal and head of the caudate infarcts were found in the other two cases respectively [10]. In a larger series of ischemia related mania, 8 stroke patients who subsequently developed mania had right hemisphere lesions primarily in medial temporal, orbitofrontal, caudate or thalamic regions [11]. An average interval of 5.8 months elapsed between lesion onset and secondary manic symptoms in this cohort, highlighting the fact that secondary mania can occur in the immediate post-stroke period or following a temporal delay. More recent case reports also implicate orbitofrontal, medial temporal and subcortical structures [30-34]. The diversity of identified lesions in secondary mania has been framed in the context of well-described prefrontal-subcortical circuits, with a particular association to the orbitofrontal-subcortical network involved in empathic, socially appropriate behavior [35-37]. The orbitofrontal cortex projects to the ventral medial caudate, which in turn connects to the dorsal medial globus pallidus interna (GPi). The GPi projects to the ventral anterior and dorsomedial thalamic nuclei, and the direct cortical-subcortical loop is completed by thalamic projections to the orbitofrontal cortex (Figure 2). Distinct fiber tracks also connect the orbitofrontal cortex with medial temporal lobe structures [38].

The multiplicity of lesions implicated in secondary mania has also been explained, in part, through the concept of diaschisis; lesions in one area of the brain affect function in spatially remote regions. Advanced

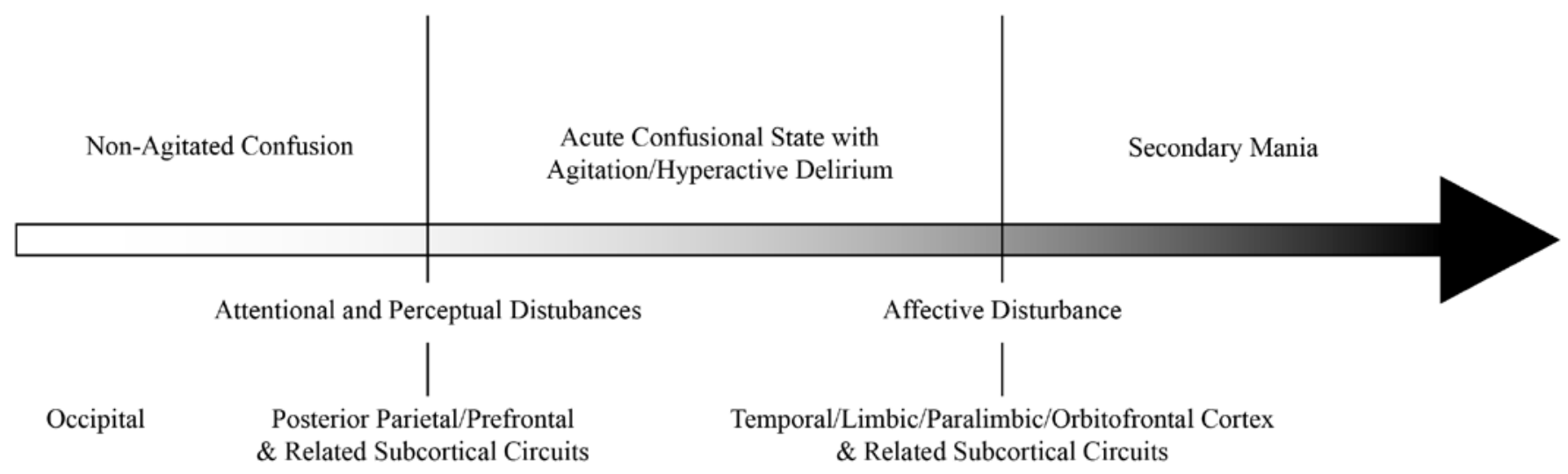

Figure 3: Right hemispheric lesions associated with non-agitated confusion, hyperactive delirium, and secondary mania. Agitated and non-agitated confusional states share overlapping cortical and subcortical neural substrates including posterior parietal, prefrontal, and related subcortical connections. Hyperactive delirium and secondary mania share overlapping cortical and subcortical neural substrates including medial temporal and paralimbic cortices. Secondary mania is particularly associated with orbitofrontal-subcortical circuit lesions. 
functional neuroimaging techniques have refined this concept further by detailing functional connectivity across large-scale brain networks [39]. In early work on secondary mania contrasting 5 patients with mania after cortical lesions with 3 manic patients following subcortical lesions (with varying durations of post-stroke onset), patients with only subcortical lesions exhibited additional, spatially remote, medial temporal hypometabolism seen on ${ }^{18}$ flourodeoxyglucose positron emission tomography $\left({ }^{18} \mathrm{FDG}-\mathrm{PET}\right)[40] .2$ of these 3 patients with subcortical lesions had ischemic strokes within the anterior limb of the internal capsule and caudate. In related investigations, a patient with bilateral dorsomedial thalamic infarcts and subacute secondary hypomania exhibited non-lesional hypoperfusion on a single-positron emission computed tomography (SPECT) scan in right orbitofrontal cortex [41]. A case of mania with delirium occurring within 24 hours after a right paramedian thalamic stroke similarly showed remote nonlesional hypoperfusion in right frontal cortex on a post-stroke SPECT scan [24]. In a unique case, a 78-year-old woman hospitalized for major depression suffered a right MCA stroke and acutely developed mania [42]. Compared to a fortuitously obtained pre-stroke SPECT scan, a post-stroke scan showed relative hypoperfusion of the right orbitofrontal, medial frontal, and dorsolateral frontal cortices and unexpected hyperperfusion of the left orbitofrontal cortex. This case helped detail a possible role for post-stroke intrahemispheric disconnection in the biology of secondary mania; right hemispheric hypoactivity and reduced transcallosal inhibition may result in subsequent left hemispheric hyperactivity and ensuing secondary mania.

\section{The spectrum of confusion, hyperactive delirium, and secondary mania}

Having discussed right hemispheric stroke lesions associated with non-agitated confusion, hyperactive delirium, and secondary mania, the neural substrates of these phenomena may be viewed as having both distinct and overlapping components (Figure 3). Non-agitated confusional states may preferentially involve higher order perceptual/ attentional association cortices (parietal, occipital, prefrontal) and related subcortical connections. Hyperactive delirium overlaps with these sites but has a tendency to also involve limbic related medial temporal lobe structures which may augment the salience of perceptual and attentional disturbances through impaired regulation of sympathetic outflow. Lesions associated with secondary mania more commonly involve orbitofrontal-subcortical circuits and medial temporal lobe structures, although orbitofrontal-subcortical lesions are not specific for secondary mania, and similar abnormalities may manifest with impulsivity in the absence of frank mania [37].

\section{Other factors}

It is important to note that while in this article the neuroanatomical correlates of non-agitated confusion, hyperactive delirium, and secondary mania are reviewed in the context of right hemispheric strokes, non-right hemispheric lesions have also been associated with these disturbances. One study of 155 acute cerebrovascular accidents, including both ischemic and hemorrhagic stroke, suggested that acute confusional states were more common with left-sided hemispheric lesions [5], a finding also reported in additional case-based observations [43]. Similarly, studies examining the neural correlates of disorientation and mania have not consistently identified a right hemispheric lateralization [44,45,12,46-48]. Brainstem lesions have also been associated with similar behavioral and affective disturbances, including reports of ventral pontine infarcts presenting acutely with secondary mania (presumably in the setting of disruptions to ascending reticular activating and monoamine pathways) [49].

While this article focuses on lesion localization, additional factors appear to confer increased vulnerability to the development of confusion, hyperactive delirium, and mania in the setting of right hemisphere lesions. These include factors resulting in more global dysfunction as well as inherent genetic predispositions. For example, development of post-stroke acute confusional states with or without agitation is associated with pre-existing cognitive decline [50]. Vascular risk factors also correlate with the development of secondary mania. Fujikawa and colleagues in 1995 compared 20 patients with late onset mania and 20 patients with early onset mania, and found that $65 \%$ of late-onset mania patients had silent cerebral infarcts compared with only $25 \%$ of early-onset mania patients [51]. Small vessel risk factors including hypertension, hyperlipidemia, and diabetes mellitus are associated with late life onset mania [52], and subcortical microangiopathic disease has been linked to delirium [53]. Starkstein, Robinson, and coworkers in 1988 suggested that a confluence of factors - the combination of right lateralized limbic and paralimbic lesions and either a family history of affective disorders or frontal subcortical atrophy - predispose to the development of secondary mania following stroke. This was well detailed in a comparative study of 11 patients who developed mania following brain injury compared to 25 patients with idiopathic mania (bipolar disorder), 11 lesion-matched controls without mania, and 11 healthy normal controls [10]. Patients with secondary mania had focal brain lesions involving right hemisphere predominant, limbic related regions and either had frontal subcortical atrophy predating their stroke or a family history of affective disorders in $1^{\text {st }}$ degree relatives. Regarding prognosis, evidence suggests that poststroke delirium is associated with increased morbidity and mortality $[5,53]$, but there is little to no controlled data to date, apart from casebased observations, to inform prognosis in patients with secondary mania.

\section{Conclusion}

In summary, non-agitated confusion, hyperactive deliriums, and secondary mania, when considered as a group, are frequently encountered behavioral and affective disturbances in patients with right hemispheric strokes. The reported case of a gentleman with vascular risk factors presenting with confusion and agitation in the context of a subacute right MCA infarct with associated microvascular disease and cerebral atrophy provides an example of these disturbances. Published observational studies suggest preferential involvement of higher order perceptual-attentional related ischemic lesions in confused patients with or without agitation. Limbic and paralimbic cortical-subcortical circuits are more frequently compromised in patients with hyperactive delirium or secondary mania. Specific orbitofrontal-subcortical and medial temporal lesions are frequently implicated in secondary mania. Given that many of the studies to date on these neuropsychiatric syndromes are retrospective and primarily descriptive in nature, further investigations using more recently developed advanced structural and functional neuroimaging techniques, including automated volumetric and functional connectivity analyses, are needed to provide added specificity to the delineation of structure-function relationships in these patient populations.

\section{References}

1. Ropper $A H$, Adams RD, Victor M, Samuels MA, Ropper AHA, et al. (2009) Adams and Victor's principles of neurology. McGraw-Hill Medical, New York. 
2. Ferro JM, Caeiro L, Verdelho A (2002) Delirium in acute stroke. Curr Opin Neurol 15: 51-55.

3. McManus J, Pathansali R, Stewart R, Macdonald A, Jackson S (2007) Delirium post-stroke. Age Ageing 36: 613-618.

4. Meagher DJ, Trzepacz PT (2000) Motoric subtypes of delirium. Semin Clin Neuropsychiatry 5: 75-85

5. Gustafson Y, Olsson T, Eriksson S (1991) Acute confusional states (delirium) in stroke patients. Cerebrovasc Dis: 257-265.

6. Langhorne P, Stott DJ, Robertson L, MacDonald J, Jones L, et al. (2000) Medical complications after stroke: a multicenter study. Stroke 31: 1223-1229.

7. Caeiro L, Ferro JM, Albuquerque R, Figueira ML (2004) Delirium in the first days of acute stroke. J Neurol 251: 171-178.

8. Krauthammer C, Klerman GL (1978) Secondary mania: manic syndromes associated with antecedent physical illness or drugs. Arch Gen Psychiatry 35: 1333-1339.

9. Association AP (2000) Diagnostic and statistical manual of mental disorders : DSM-IV-TR. American Psychiatric Association, Washington, DC.

10. Starkstein SE, Pearlson GD, Boston J, Robinson RG (1987) Mania after brain injury. A controlled study of causative factors. Archives of neurology 44: 10691073.

11. Robinson RG, Boston JD, Starkstein SE, Price TR (1988) Comparison of mania and depression after brain injury: causal factors. The American journal of psychiatry 145: $172-178$

12. House A, Dennis M, Warlow C, Hawton K, Molyneux A (1990) Mood disorders after stroke and their relation to lesion location. A CT scan study. Brain 113 1113-1129.

13. Burvill PW, Johnson GA, Jamrozik KD, Anderson CS, Stewart-Wynne EG, et al. (1995) Prevalence of depression after stroke: the Perth Community Stroke Study. Br J Psychiatry 166: 320-327.

14. Schmidley JW, Messing RO (1984) Agitated confusional states in patients with right hemisphere infarctions. Stroke 15: 883-885.

15. Robinson RG, Kubos KL, Starr LB, Rao K, Price TR (1984) Mood disorders in stroke patients. Importance of location of lesion. Brain 107: 81-93.

16. Shapiro BE, Danly M (1985) The role of the right hemisphere in the control of speech prosody in propositional and affective contexts. Brain Lang 25: 19-36.

17. Starkstein SE, Fedoroff JP, Price TR, Leiguarda R, Robinson RG (1993) Apathy following cerebrovascular lesions. Stroke 24: 1625-1630.

18. Horenstein S, Chamberlin W, Conomy J (1967) Infarction of the fusiform and calcarine regions: agitated delirium and hemianopia. Transactions of the American Neurological Association 92: 85-89.

19. Faris AA (1969) Limbic system infarction: a report of two cases. Neurology 19:91-96.

20. Mesulam MM, Waxman SG, Geschwind N, Sabin TD (1976) Acute confusiona states with right middle cerebral artery infarctions. J Neurol Neurosurg Psychiatry 39: 84-89.

21. Mullally W, Huff K, Ronthal M, Geschwind N (1989) Chronic confusional state with right middle cerebral artery occlusion. N J Med 32: 541-544.

22. Mullally W, Huff J, Ronthal M, Geschwind N (1982b) Frequency of acute confusional states with lesions of the right hemisphere. Annals of neurology 12: 113

23. Caplan LR, Schmahmann JD, Kase CS, Feldmann E, Baquis G, et al. (1990) Caudate infarcts. Archives of neurology 47: 133-143.

24. Bogousslavsky J, Ferrazzini M, Regli F, Assal G, Tanabe H, et al. (1988) Manic delirium and frontal-like syndrome with paramedian infarction of the right thalamus. J Neurol Neurosurg Psychiatry 51: 116-119.

25. Mori E, Yamadori A (1987) Acute confusional state and acute agitated delirium. Occurrence after infarction in the right middle cerebral artery territory. Archives of neurology 44: 1139-1143.

26. Caplan LR, Kelly M, Kase CS, Hier DB, White JL, et al. (1986) Infarcts of the inferior division of the right middle cerebral artery: mirror image of Wernicke's aphasia. Neurology 36: 1015-1020.

27. Fisher CM (1982) Disorientation for place. Archives of neurology 39: 33-36.
28. Starkstein SE, Robinson RG, Price TR (1987) Comparison of cortical and subcortical lesions in the production of poststroke mood disorders. Brain 110 1045-1059.

29. Huffman J, Stern TA (2003) Acute psychiatric manifestations of stroke: a clinical case conference. Psychosomatics 44: 65-75.

30. Goyal R, Sameer M, Chandrasekaran R (2006) Mania secondary to right-sided stroke--responsive to olanzapine. General Hospital Psychiatry 28: 262-263.

31. Nagaratnam N, Wong K, Patel I (2006) Secondary mania of vascular origin in elderly patients: A report of two clinical cases. Archives of Gerontology and Geriatrics 43: 223-232.

32. da Rocha FF, Correa H, Teixeira AL (2008) A successful outcome with valproic acid in a case of mania secondary to stroke of the right frontal lobe. Progress in neuro-psychopharmacology biological psychiatry 32: 587-588

33. Dervaux A, Levasseur M (2008) Risperidone and Valproate for Mania Following Stroke. J Neuropsychiatry Clin Neurosci 20: 247.

34. Alexander GE, DeLong MR, Strick PL (1986) Parallel organization of functionally segregated circuits linking basal ganglia and cortex. Annu Rev Neurosci 9: 357-381.

35. Cummings JL (1993) Frontal-subcortical circuits and human behavior. Arch Neurol 50: 873-880.

36. Mega MS, Cummings JL (1994) Frontal-subcortical circuits and neuropsychiatric disorders. J Neuropsychiatry Clin Neurosci 6: 358-370.

37. Carmichael ST, Price JL (1995) Limbic connections of the orbital and media prefrontal cortex in macaque monkeys. J Comp Neurol 363: 615-641.

38. Fox MD, Raichle ME (2007) Spontaneous fluctuations in brain activity observed with functional magnetic resonance imaging. Nat Rev Neurosci 8: 700-711.

39. Starkstein SE, Mayberg HS, Berthier ML, Fedoroff P, Price TR, et al. (1990) Mania after brain injury: neuroradiological and metabolic findings. Annals of neurology 27:652-659

40. Benke T, Kurzthaler I, Schmidauer C, Moncayo R, Donnemiller E (2002) Mania caused by a diencephalic lesion. Neuropsychologia 40: 245-252.

41. Mimura M, Nakagome K, Hirashima N, Ishiwata H, Kamijima K, et al. (2005) Left frontotemporal hyperperfusion in a patient with post-stroke mania. Psychiatry research 139: 263-267.

42. Devinsky O, Bear D, Volpe BT (1988) Confusional states following posterio cerebral artery infarction. Arch Neurol 45: 160-163.

43. Jampala VC, Abrams R (1983) Mania secondary to left and right hemisphere damage. The American journal of psychiatry 140: 1197-1199.

44. Starkstein SE, Boston JD, Robinson RG (1988) Mechanisms of mania after brain injury. 12 case reports and review of the literature. The Journal of nervous and mental disease 176: 87-100.

45. Desmond DW, Tatemichi TK, Figueroa M, Gropen TI, Stern Y (1994) Disorientation following stroke: frequency, course, and clinical correlates. J Neurol 241: 585-591.

46. Pedersen PM, Jorgensen HS, Nakayama H, Raaschou HO, Olsen TS (1998) Impaired orientation in acute stroke: frequency, determinants, and time-course of recovery. The Copenhagen Stroke Study. Cerebrovasc Dis 8: 90-96.

47. Fenn D, George K (1999) Post-stroke mania late in life involving the left hemisphere. Aust N Z J psychiatry 33: 598-600.

48. Drake ME, Pakalnis A, Phillips B (1990) Secondary mania after ventral pontine infarction. The Journal of neuropsychiatry and clinical neurosciences 2: 322 325

49. Henon H, Lebert F, Durieu I, Godefroy O, Lucas C, et al. (1999) Confusiona state in stroke: relation to preexisting dementia, patient characteristics, and outcome. Stroke 30: 773-779.

50. Fujikawa T, Yamawaki S, Touhouda Y (1995) Silent cerebral infarctions in patients with late-onset mania. Stroke 26: 946-949.

51. Cassidy F, Carroll BJ (2002) Vascular risk factors in late onset mania. Psychological medicine 32: 359-362.

52. Soiza RL, Sharma V, Ferguson K, Shenkin SD, Seymour DG, et al. (2008) Neuroimaging studies of delirium: a systematic review. J Psychosom Res 65 239-248.

53. Sheng AZ, Shen Q, Cordato D, Zhang YY, Yin Chan DK (2006) Delirium within three days of stroke in a cohort of elderly patients. J Am Geriatr Soc 54: 1192 1198. 